\title{
Nahrungsmittelunverträglichkeiten - Update
}

\author{
Stephan C. Bischoff
}

\begin{tabular}{|l|r|}
\hline Übersicht & \\
\hline Definitionen & 143 \\
Epidemiologie & 144 \\
Pathophysiologie & 145 \\
Klinisches Bild & 149 \\
Diagnostik & 152 \\
Therapie & 155 \\
\hline
\end{tabular}

\section{Definitionen}

Hypersensitivitätsreaktionen. Nahrungsstoffe, insbesondere Nahrungsproteine, sind unverzichtbarer Bestandteil unseres Lebens und müssen regelmäßig zugeführt werden. Andererseits können Nahrungsstoffe krankheitsauslösend werden, wenn sie vom Immunsystem des Gastrointestinaltrakts fälschlicherweise als schädliche Agenzien betrachtet werden und entsprechende immunologische Reaktionen auslösen. Solche aberranten immunologischen Reaktionen verursachen Autoimmunerkrankungen und Allergien, die sich per definitionem zunächst dadurch unterscheiden, dass das auslösende Antigen entweder endogen synthetisiert oder exogen zugeführt wird. Beide Formen aberranter Immunreaktionen werden unter dem Begriff „Hypersensitivitätsreaktionen“ zusammengefasst, die nach Coombs und Gell in 4 Formen klassifiziert werden (s. „Klassifikation“ und Tab. 1) [1].

Die Konsequenz solcher Hypersensitivitätsreaktionen ist in aller Regel eine pathologische Entzündungsreaktion, die je nach Ausmaß und Dauer die Funktion einzelner Organe und im Extremfall des gesamten Organismus beeinträchtigt. Nahrungsproteine, die solche allergischen Reaktionen auslösen, werden Nahrungsmittelallergene genannt (abgeleitet von den griechischen Wörtern „allos“" anders und „ergon“ = Arbeit).

\section{Klassifikation}

\section{Hypersensitivitätsreaktionen nach Coombs und Gell}

Die Engländer Coombs und Gell haben 1963 eine Einteilung der pathologischen Immunreaktionen in 4 Formen in Abhängigkeit vom antigenerkennenden Molekül vorgenommen. Die Typ-I-Reaktion ist eine Sofortreaktion, der eine ver- zögerte Reaktion folgen kann („late phase reaction“), die schwer von Typ-IV-Reaktionen unterscheidbar ist. Die Typ-III-Reaktion kann ebenfalls als Sofortreaktion verlaufen und ist dann schwer von der Typ-ISofortreaktion zu unterscheiden.

\section{Tabelle 1}

\section{Hypersensitivitätsreaktionen nach Coombs und Gell.}

\begin{tabular}{|c|c|c|}
\hline Typ & $\begin{array}{l}\text { Antigenerkennung } \\
\text { durch }\end{array}$ & Klinische Beispiele \\
\hline $\mathrm{I} a / b$ & $\lg E$ & $\begin{array}{l}\text { atopische Erkrankungen, Asthma, Allergien in- } \\
\text { klusive Nahrungsmittelallergien vom Soforttyp }\end{array}$ \\
\hline II & $\lg G, \lg M$ & $\begin{array}{l}\text { hämolytische Transfusionsreaktion, idiopathische } \\
\text { Thrombozytopenie, Agranulozytose }\end{array}$ \\
\hline III a/b & IgG-Immunkomplexe & $\begin{array}{l}\text { exogen allergische Alveolitis, allergische Vasku- } \\
\text { litis, Serumkrankheit }\end{array}$ \\
\hline IV & T-Lymphozyten & $\begin{array}{l}\text { Kontaktallergie, Tuberkulin-Reaktion, Hypersen- } \\
\text { sitivitätspneumonitis }\end{array}$ \\
\hline
\end{tabular}


Abb. 1 Klassifikation von Nahrungsmittelunverträglichkeiten nach den zugrunde liegenden Mechanismen.

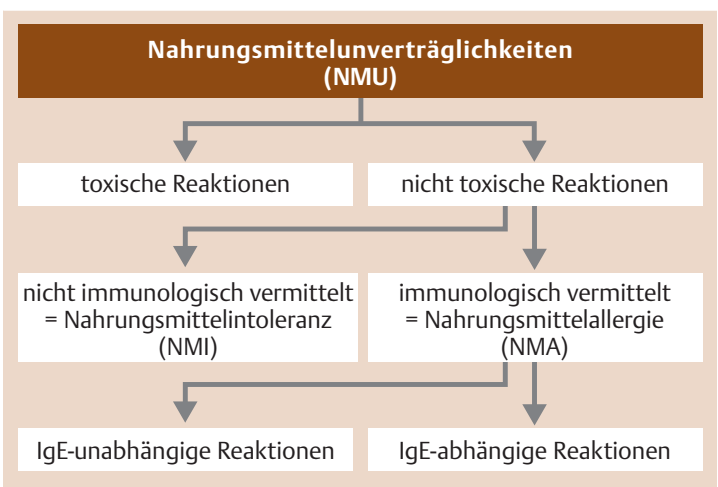

Nahrungsmittelallergien und -intoleranzen. Nahrungsmittelallergien (NMA) und Nahrungsmittelintoleranzen (NMI) sind zwei Unterformen von abnormalen Reaktionen auf Nahrungsmittel (,adverse reactions to food“, ARF; oder Nahrungsmittelunverträglichkeiten, NMU), wobei NMA eine Immunpathogenese zugrunde liegt, während NMI auf anderen Pathomechanismen beruhen (Abb. 1). NMI sind beispielsweise die Laktoseintoleranz, die Histaminintoleranz oder Pseudoallergien, die durch unspezifische Mastzellaktivatoren ausgelöst werden.

NMA manifestieren sich je nach Altersstufe in bis zu $50 \%$ vor allem in Form von gastrointestinalen (GI) Symptomen. Deshalb konsultieren viele dieser Patienten den Gastroenterologen, der in den meisten Fällen nicht ausreichend mit dem Krankheitsbild vertraut ist $[2,3]$. Häufig werden die Patienten dann als „psychosomatisch“ deklariert oder die Beschwerden als „funktionell“ oder „Reizdarmsyndrom“ (RDS) klassifiziert, ohne das tatsächliche Problem zu definieren. Andererseits ist bekannt, dass RDS und NMU häufig miteinander assoziiert sind und dass die NMA manchmal ein zugrunde liegender Mechanismus für Symptome bei einer Subgruppe von Patienten mit RDS sind $[4,5]$. Das Thema NMA wurde zusätzlich relevant durch die Erkenntnis, dass durch Nahrungsmittel ausgelöste allergische Reaktionen die häufigste Ursache für lebensbedrohliche Anaphylaxien sind [6].

Nahrungsmittelallergien (NMA) sind definiert als individuell auf bestimmte Nahrungsmittel auftretende Nahrungsmittelunverträglichkeiten (NMU), denen eine Immunpathogenese zugrunde liegt, während Nahrungsmittelintoleranz (NMI) ein Sammelbegriff für nicht immunologisch bedingte NMU ist.

\section{Epidemiologie}

NMA treten besonders häufig im frühen Kindesalter auf und verschwinden meist spontan mit zunehmendem Alter bis zur Einschulung. Manchmal werden die frühkindlichen NMA abgelöst von Allergien gegen inhalative Allergene wie Pollen, Milben oder Tierepithelien. Nur wenige Kinder behalten ihre NMA bis zum Erwachsenenalter. Auf der anderen Seite gibt es Erwachsene, die an NMA leiden, ohne in der Kindheit betroffen gewesen zu sein. Die Prävalenz der NMA beträgt $4-8 \%$ bei Kindern und $1-2 \%$ bei Erwachsenen [7 - 9] (s. „Studienergebnisse - Prävalenz der NMA“). Dagegen steht, dass etwa 20\% aller Erwachsenen in Industrienationen über Nahrungsmittelunverträglichkeiten klagen $[3,10]$. Die Mehrzahl der Fälle von NMU ist nicht immunologisch vermittelt, wobei die Laktoseintoleranz die häufigste Form in westlichen Ländern darstellt.

\section{Studienergebnisse}

\section{Prävalenz der NMA}

Die Daten zur Prävalenz von NMA wurden in neueren Metaanalysen bestätigt und präzisiert [11, 12]. Trotz heterogener Studienlage ließ sich eine „self-reported“ Prävalenz von 1,2 - 17\% für Milch, 0,2 - 7\% für Ei, $0-2 \%$ für Erdnuss und für Fisch, 0 - $10 \%$ für Meeresfrüchte und 3-35\% für irgendein Nahrungsmittel eruieren [11]. Wenn man Provokationstests zugrunde legt, zeigt sich eine Prävalenz von 0,1-4,3\% für Früchte und Nüsse, $0,1-1,4 \%$ für Gemüse und $<1 \%$ für Weizen, Soja und Sesam [12]. Dabei wurden vorwiegend Studien mit Kindern und Erwachsenen aus dem angloamerikanischen Sprachraum berücksichtigt. Interessanterweise war für Obst und Gemüse die Sensibilisierungsrate niedriger als die subjektive Wahrnehmung der Allergie, während es für Weizen und Soja gerade umgekehrt war [12].

Hygiene und immunologische Erkrankungen. Es ist anzunehmen, dass analog zur Entwicklung anderer allergischer Erkrankungen im Allgemeinen auch NMA in den letzten Jahrzehnten deutlich zugenommen haben. Allerdings liegen konkrete Zahlen, die eine Zunahme der Prävalenz von NMA belegen, nur für die Erdnussallergie vor [13]. Die Gründe für eine solche Zunahme sind nicht vollkommen klar, wenngleich neuere epidemiologische Studien vermuten lassen, dass ein gesteigerter Hygienestatus in den Industrienationen mit einer gesteigerten Allergieinzidenz assoziiert ist $[14,15]$. Es konnte gezeigt werden, dass ausrei- 
chende Exposition mit bakteriellem Lipopolysaccharid (LPS) in den ersten Lebensjahren einen protektiven Effekt hinsichtlich der Entwicklung allergischer Erkrankungen haben könnte [16]. Diese Studien, die aus der „Hygienetheorie“ heraus entwickelt wurden (s. „Forschungsergebnisse-Hygienetheorie“), deuten darauf hin, dass für die Ausbildung allergischer Erkrankungen nicht nur das spezifische, sondern auch das angeborene Immunsystem von zentraler Bedeutung ist. Die Hygienetheorie bezieht sich auf die zunehmende Inzidenz nicht nur von allergischen Erkrankungen, sondern auch von anderen immunologischen Erkrankungen wie rheumatischer Arthritis, Typ-1-Diabetes mellitus und chronisch entzündlichen Darmerkrankungen [16].

Bei Kindern beträgt die Prävalenz der NMA 4-8\%, bei Erwachsenen 1-2\%. In Industrienationen geben etwa $20 \%$ aller Erwachsenen NMU an, wobei die NMI, deren häufigste Form in westlichen Ländern die Laktoseintoleranz darstellt, dominieren.

\section{Forschungsergebnisse}

\section{Hygienetheorie}

Die Hygienetheorie besagt, dass eine ausgeprägt hygienische Umgebung mit reduzierter mikrobieller Exposition und konsekutiv reduziertem Einfluss von Th1-Zytokinen einen Risikofaktor darstellt für die Entwicklung von Th2-dominierten immunologischen Erkrankungen wie Allergien oder auch Autoimmunerkrankungen, zu denen der Typ-1-Diabetes, die chronische Polyarthritis und auch chronisch entzündliche Darmerkrankungen gehören. Die Theorie gründete sich zunächst auf epidemiologische Beobachtungen und wurde inzwischen experimentell sowie klinisch verifiziert.

\section{Pathophysiologie}

\section{Immunpathogenese allergischer Erkrankungen}

Abhängig vom antigenerkennenden Molekül (IgE, IgG, Immunkomplexe, T-Zell-Rezeptor) werden immunologische Hypersensitivitätsreaktionen nach Coombs und Gell in die Typen I-IV eingeteilt [1].

IgE-vermittelte Reaktion. Die am besten charakterisierte Überempfindlichkeitsreaktion gegen Nahrungsmittel ist die IgE-vermittelte Typ-I-Reaktion, die auch vie-

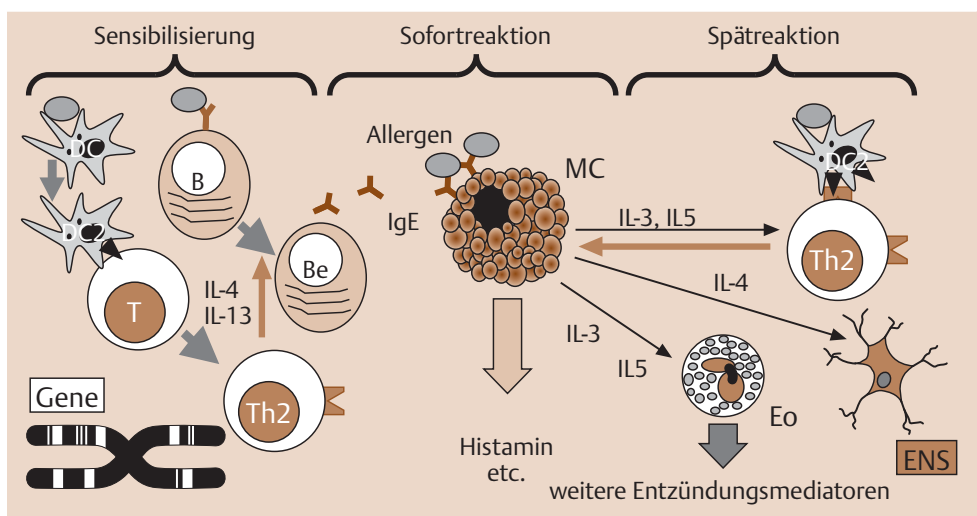

Abb. 2 Phasen der allergischen Reaktion. DC: dendritische Zellen, DC2: DC Typ 2, T: T-Lymphozyten, Th2: T-Helfer-Lymphozyten Typ 2, B: B-Lymphozyten, Eo: Eosinophile, MC: Mastzellen, ENS: enterisches Nervensystem.

len Fällen von Asthma bronchiale, saisonaler Rhinitis, Urtikaria und atopischem Ekzem zugrunde liegt. Manche Menschen entwickeln nach der akuten IgE-Reaktion eine verzögerte Reaktion, die durch eine erhöhte zelluläre Infiltration des betroffenen Gewebes mit Entzündungszellen und schließlich durch eine Gewebedysfunktion gekennzeichnet ist (Abb. 2). Solche Mechanismen spielen sowohl bei milch- und sojaproteininduzierten Enteropathien als auch bei der Zöliakie eine Rolle. Immunologischen Reaktionen gegen Nahrungsmittel können auch gemischte IgE-abhängige und IgE-unabhängige Reaktionen zugrunde liegen. Insbesondere bei Typ-IV-Hypersensitivitätsreaktionen gegen Nahrungsproteine wird vermutet, dass sie auf der Präsenz von nahrungsmittelantigenspezifischen T-HelferZellen und zytotoxischen T-Zellen beruhen $[3,17,18]$.

Allergische Reaktionen auf Nahrungsmittel sind meist IgE-vermittelt (Typ-I-Sofortreaktionen), es treten aber auch verzögerte Reaktionen (Late-Phase-Typ-1- oder Typ-IV-Reaktionen) auf.

\section{- Voraussetzungen für die Entwicklung einer allergischen Reaktion}

Für die Entwicklung einer allergischen Darmentzündung werden eine ausreichende Menge an Allergen im Darm und ein hyperreagibles mukosales Immunsystem benötigt. Der erhöhten Antigenexposition kann eine genetisch determinierte Veränderung von Schlüsselmolekülen der Darmbarriere, eine Unreife der Darmbarriere, eine erworbene Störung des angeborenen Darmimmunsystems, beispielsweise bedingt durch eine Darminfektion, oder eine Kombination hieraus zugrunde liegen. Unspezifische Entzündung, ausgelöst durch Bakterien, Viren oder Toxine, kann den Verlust der immunologischen Toleranz und die nachfolgende 
Entwicklung einer Hypersensitivität des mukosalen Immunsystems gegen luminale Antigene triggern. Ähnliche Mechanismen wurden für andere Entzündungserkrankungen des GI-Trakts wie chronisch entzündliche Darmerkrankungen (CED) beschrieben, wobei dort eher Bakterienantigene als Nahrungsantigene Auslöser zu sein scheinen [19].

IgA- und IgE-Produktion. Eine verzögerte Entwicklung des protektiven IgA-Systems innerhalb des „gut-associated lymphoid tissue“ (GALT) in der postnatalen Phase oder ein gesteigerter Switch hin zu IgE-produzierenden B-Lymphozyten ist mit einem erhöhten Risiko für die Entwicklung von allergischen Erkrankungen assoziiert. Die IgA-Synthese wird hauptsächlich durch TGF- $\beta$ aus Th3-Zellen und externe Trigger induziert, während die IgE-Synthese abhängig ist von CD40Ligand sowie den Zytokinen IL-4 und IL-13, die von Th2-Zellen und Entzündungszellen (Mastzellen, Basophile) produziert werden [17]. Dagegen inhibieren Th1-Zytokine wie IFN- $\gamma$ die Aktivität von Th2-Zellen, was erklärt, wie eine kontrollierte Th1-dominierte Immunantwort, beispielsweise durch bestimmte Bakterienprodukte getriggert, dazu beitragen kann, eine primär vorgegebene Th2-Antwort im Darm zu limitieren und dadurch Überproduktion von IgE zu verhindern. Solche Mechanismen unterstützen die bereits erwähnte „Hygienetheorie“.

\section{- Angeborenes und spezifisches Immunsystem}

Bei immunologischen Überempfindlichkeitsreaktionen ist nicht nur das spezifische Immunsystem, sondern auch das angeborene (,innate“) Immunsystem involviert. Die Charakterisierung von Schlüsselmolekülen der angeborenen Immunabwehr wie Defensine, Mucine oder Synactin und ihrer möglichen Veränderungen bei Allergikern ist deshalb für das Verständnis der Mechanismen und die Entwicklung neuer Therapiekonzepte von zentraler Bedeutung [20]. Störungen innerhalb des angeborenen Immunsystems können auch für Deviationen des spezifischen Immunsystems verantwortlich sein, die beispielsweise in die Überproduktion von spezifischem IgE münden.

Th2-Zytokine. Im Verlauf der allergischen Reaktion werden naive Lymphozyten des GALT (= gut-associated lymphatic tissue) zur Bildung von Th2-Zytokinen wie IL-4 und IL-13 veranlasst, die die Entwicklung IgE-produzierender Plasmazellen fördern. Tatsächlich können allergenspezifische T-Zellen aus dem Blut, der Haut und der Mukosa von Patienten mit NMA isoliert werden, die neben IL-4 und IL-13 auch IL-5 produzieren. Diese Zytokine regulieren nicht nur die IgE-Synthese
(IL-4, IL-13), sondern auch die Einwanderung und Aktivierung von Entzündungszellen wie Mastzellen (IL-4) und eosinophilen Granulozyten (IL-5) [3, 17, 18]. In klinischen Studien konnte gezeigt werden, dass IgE lokal umschrieben in der respiratorischen und gastrointestinalen Mukosa produziert wird. Dies erklärt, warum Serum-IgE-Messungen und Hauttests nicht eng mit mukosalen allergischen Reaktionen im Darm korrelieren. Bei Atopikern sind die erhöhten IgE-Spiegel eng korreliert mit IL-13, dessen Gen einem Polymorphismus unterliegt, der mit Atopie assoziiert ist.

Drei Phasen. Die IgE-vermittelte allergische Immunantwort kann in drei Phasen eingeteilt werden:

- die klinisch stumme Sensibilisierungsphase, meist im Säuglings- oder Kindesalter,

- die symptomatische Effektorphase, die sich aus einer akuten und einer fakultativen verzögerten Reaktion zusammensetzt, und

- die chronische, organzerstörende Phase, die ein Resultat repetitiv auftretender verzögerter Reaktionen sein könnte [3].

Voraussetzung für die allergische Reaktion ist eine Sensibilisierungsphase, in der das Immunsystem auf eine pathologische Reaktion umprogrammiert wird und die begünstig wird durch eine unreife bzw. gestörte Darmbarriere.

\section{Entzündungszellen}

Entzündungsmediatoren aus Mastzellen und Eosinophilen sind primär verantwortlich für die klinischen Symptome der Patienten mit Nahrungsmittelallergie $[18,21]$. Man findet bei diesen Patienten erhöhte Spiegel von Histamin (bzw. Methylhistamin), Tryptase, eosinophilem kationischem Protein (ECP), IL-5 und TNF- $\alpha$ in Serum, Urin, Darmspülflüssigkeit und Stuhlproben $[3,22]$. Histologische Untersuchungen zeigen, dass Mastzellen und Eosinophile in der Darmmukosa nach lokaler Provokationstestung degranulieren und Mediatoren wie Zytokine freisetzen [23]. Inzwischen werden diese Zellen nicht nur als Entzündungszellen, sondern auch als immunmodulatorische Zellen verstanden, die zur Homöostase im Darm und zur Abwehr von Bakterien und Parasiten beitragen [3].

\section{Rolle des Darmnervensystems}

In den letzten Jahren wurde deutlich, dass auch das enterische Nervensystem (ENS) an der Regulation von allergischen Entzündungszellen wie Lymphozyten, Mastzellen und Eosinophilen beteiligt ist. Die morphologische funktionelle Assoziation zwischen Immunzellen und Nervenzellen wurde primär für Mastzellen 
beschrieben [24] und teilweise auf Eosinophile ausgeweitet [25]. Zu betonen ist, dass nicht nur das GALT innerviert ist, sondern auch umgekehrt das ENS durch Mediatoren des mukosalen Immunsystems entscheidend mitreguliert wird $[3,25]$. Derartige Neuroimmuninteraktionen mögen die häufigen psychologischen und funktionellen Begleitsymptome erklären, die viele Patienten mit allergischen und anderen chronischen Darmerkrankungen kennzeichnen.

An einer allergischen Reaktion in voller Ausprägung sind neben Mastzellen meist auch andere Entzündungszellen (eosinophile und basophile Granulozyten), Th2-Lymphozyten, Nervenzellen und andere Gewebezellen beteiligt.

\section{Nicht immunologische Mechanismen der NMI}

Nahrungsmittelintoleranzen sind definiert als nicht immunologisch vermittelte Nahrungsmittelunverträglichkeiten und somit zumindest pathophysiologisch klar abgegrenzt von Nahrungsmittelallergien. Auf der klinischen Ebene ist diese Abgrenzung weniger deutlich, manchmal sogar kaum möglich, weil die Symptomatik von Patienten mit NMA und NMI sich oft kaum unterscheidet. In Tab. $\mathbf{2}$ werden die wichtigsten Formen der NMI dargestellt.

Nahrungsmittelvergiftung. Die Nahrungsmittelvergiftung, ausgelöst durch Bakterien oder Bakterientoxine, ist aufgrund des kurzen Verlaufs meist anamnestisch eindeutig abgrenzbar. Chronisch infektiöse Darmerkrankungen erfordern allerdings eine eingehende mikrobiologische Untersuchung der Fäzes.

Laktoseintoleranz. Die häufigste Nahrungsmittelintoleranz bei Erwachsenen ist die Laktoseintoleranz, wobei es sich eigentlich um eine Laktosemalabsorption aufgrund mangelnder Laktase im Bürstensaum der Dünndarmepithelien handelt. Durch die reduzierte Disaccharidase und die dadurch verminderte Absorption im Dünndarm kommt es zur bakteriellen Fermentierung des Zuckers im Dickdarm mit Gasbildung und Induktion von GI-Symptomen.

Biogene Amine und Histaminintoleranz. In der Relevanz möglicherweise unterschätzt wird die Histaminintoleranz, der ebenfalls ein Enzymmangel (an Diaminooxidase) zugrunde liegt. Bei den betroffenen Individuen führen bereits geringe Mengen an Histamin (beispielsweise in Rotwein, Champagner, Käse, Fisch,

\section{Tabelle 2}

Wichtigste Formen der Nahrungsmittelintoleranz.

- Nahrungsmittelvergiftung (Bakterien bzw. Bakterientoxine)

- Laktoseintoleranz (Mangel an Laktase)

- Histaminintoleranz (Mangel an Diaminooxidase)

- Intoleranz gegen andere biogene Amine u. Ä. (Tyramin, Serotonin, Glutamat)

- „Pseudoallergien“ (unspezifische Mastzellaktivierung durch Nahrungsmittel)

- Psychologische Nahrungsmittelunverträglichkeit (z. B. nach frühkindlicher Gewalt)

- Physiologische Nahrungsunverträglichkeit (besonders bei GI-Vorerkrankungen)

Sauerkraut) zu gastrointestinalen und neurologischen („Seekrankheit“) Symptomen. Intoleranzen gegen andere biogene Amine wie Tyramin (in Schokolade, Rotwein und Käse), Serotonin (in Bananen, Walnüssen, Ananas) und Glutamat, dem Auslöser des sog. „Chinarestaurant-Syndroms“, wurden beschrieben.

Unspezifische Mastzellaktivierung. Sie wird hervorgerufen durch Nahrungsmittel, z. B. durch Erdbeeren, Zitrusfrüchte, Tomaten, Meeresfrüchte, und Nahrungszusatzstoffe wie Salizylate, Benzoat etc. und wird auch „Pseudoallergie“ genannt, weil sie klinisch kaum abgrenzbar ist von der echten Nahrungsmittelallergie.

Psychologische und physiologische NMU. Psychologische Nahrungsmittelunverträglichkeit kann auf dem Boden von frühkindlicher Gewalt („Zwang zum Essen“) bzw. von Störungen im Bereich des ZNS oder des ENS entwickelt werden. Die physiologische Nahrungsmittelunverträglichkeit, häufig ausgelöst durch Stärke in Gemüsesorten, die zu vermehrter Gasbildung führt, wird insbesondere bei Patienten mit chronischen Darmerkrankungen (z.B. CED, RDS) beschrieben.

Die wichtigsten Formen von NMI sind die Laktoseintoleranz, die Histaminintoleranz, die Intoleranz gegen andere biogene Amine und Nahrungszusatzstoffe (Glutamat) sowie gegen Nahrungsmittel, die eine unspezifische Mastzellaktivierung auslösen können, und gegen Nahrungsstärke (besonders bei CED). 


\section{Eigenschaften von Nahrungsmittel- allergenen}

Die strukturellen und biochemischen Eigenschaften der auslösenden Allergene bestimmen die Art der Immunantwort bei der Nahrungsmittelallergie. Allerdings sind die Voraussetzungen dafür, dass ein Antigen als Allergen fungiert, bis heute unklar. Dieses Thema ist insbesondere auch für die Voraussage, ob ein Nahrungsmittel wahrscheinlich als Allergen wirkt, bzw. für die Testung neuer Nahrungsmittel auf Allergenpotenzial von zentraler Bedeutung.

Biochemische Eigenschaften und Dosis. Generell sind lösliche Proteine eher tolerogen als partikuläre oder globuläre Proteine. Andere biochemische Eigenschaften bestimmen den Absorptionsgrad und die Stabilität des Allergens im Darm. Beispielsweise ist das Erdnussprotein Ara h1 höchst resistent gegen Degradation im GI-Trakt aufgrund der Bildung von stabilen Homodimeren, was zur besonderen Relevanz dieser Form von Nahrungsmittelallergie wesentlich beiträgt. Auch die Allergendosis spielt eine Rolle, denn es konnte gezeigt werden, dass niedrige Dosen eher regulatorische T-Zellen (Th3) aktivieren, während hohe Dosen zu deren Anergie oder Apoptose führen [17].

Wichtige Allergene. Obwohl viele Nahrungsproteine als Allergene fungieren können, werden tatsächlich 90\% der NMA durch einige wenige Nahrungsmittel ausgelöst. Die „Hitliste der Nahrungsallergene“ ist abhängig von den Ernährungsgewohnheiten, die in verschiedenen Altersstufen (Säugling, Kind, Erwachsene) und in verschiedenen kulturellen Kreisen z.T. erheblich variieren können. In Tab. 3 sind die in Deutschland wichtigsten Allergene zusammengestellt.

Lösliche, leicht degradierbare Proteine sind eher tolerogen als partikuläre oder globuläre Proteine. $90 \%$ der NMA werden durch maximal 10 Nahrungsmittelsorten ausgelöst.

Kreuzreaktionen. Von besonderer Bedeutung sind die Kreuzreaktionen zwischen unterschiedlichen Nahrungsallergenen aus ähnlichen botanischen Familien und insbesondere zwischen Nahrungsallergenen und Pollen-, Milben- oder Latexallergenen [26, 27]. Kenntnis über solche Kreuzallergien ist hilfreich für die gezielte Anamnese, Diagnostik und Eliminationsdiät; außerdem erlaubt sie neue Einblicke in die „funktionelle Anatomie“ der Allergenmoleküle.

\section{Tabelle 3}

Relevante Nahrungsmittelallergene in Deutschland Quelle: „Weißbuch - Allergie in Deutschland“. Ring J, Wenning J, Hrsg. München: Urban Vogel; 2000).

\begin{tabular}{|llll|}
\hline Erwachsene & \multicolumn{3}{l}{ Kinder } \\
\hline Obst & $35 \%$ & Milch & $70 \%$ \\
\hline Nüsse & $23 \%$ & Ei & $40 \%$ \\
\hline Gewürze & $18 \%$ & Obst & $8 \%$ \\
\hline Fisch etc. & $10 \%$ & Nüsse & $5 \%$ \\
\hline Zerealien & $7 \%$ & Fisch & $5 \%$ \\
\hline Milch & $7 \%$ & Zerealien & $4 \%$ \\
\hline Ei & $4 \%$ & & \\
\hline
\end{tabular}

Rekombinante Allergene. Da es in den letzten Jahren gelang, mehr als 1000 Epitope, einschließlich etwa 50-100 Major-Epitope (s. „Forschungsergebnisse Major-Epitope“), zu klonieren und sequenzieren, sind nun rekombinante Allergene verfügbar, die neue Möglichkeiten für die Diagnostik und Therapie von allergischen Erkrankungen eröffnen. Rekombinante Allergene können für Hauttests und In-vitro-Tests anstelle von herkömmlichen Allergenextrakten eingesetzt werden, die häufig unzureichend rein und stabil sind. Zweitens können rekombinante Allergene so modifiziert werden, dass sie von T-Zellen, aber nicht von B-Zellen, erkannt werden. Dies ermöglicht grundsätzlich eine gezieltere und sicherere Desensibilisierung, was derzeit in klinischen Studien getestet wird [28].

Die Kenntnis von Kreuzreaktionen zwischen Nahrungsallergenen aus ähnlichen botanischen Familien und zwischen Nahrungsallergenen und Pollen-, Milben- oder Latexallergenen hilft bei der gezielten Anamnese und Diagnostik der NMA. 


\section{Forschungsergebnisse}

\section{Major-Epitope}

Im letzten Jahrzehnt sind sog. „Major-Allergene“ bzw. „Major-Epitope“ molekular charakterisiert worden, die sich innerhalb verwandter Nahrungsallergengruppen, aber auch in Nahrungsmitteln sowie in Pollen etc. finden und das Phänomen Kreuzallergien erklären helfen. Die ersten Major-Epitope, die kloniert wurden, waren Bet v1 und Bet v2 (Profilin), das in Birkenpollen und in zahlreichen Nahrungsmitteln wie Obst und Sellerie vorkommt. Spezifisches IgE von Patienten mit Allergie gegen Birkenpollen und Nahrungsmittel richtet sich hauptsächlich gegen Bet v1, was die Bedeutung dieser Allergenstruktur als HauptB-Zell-Epitop und IgE-Epitop für die Mastzellaktivierung unterstreicht. Seither wurden mehr als 1000 Epitope, darunter etwa 50 - 100 Major-Epitope, kloniert und sequenziert (Details unter http://www. allergome.org).

\section{Klinisches Bild}

\section{Synopsis Nahrungsmittelallergie}

Allergische Symptome variieren von geringfügigen Beeinträchtigungen bis hin zu lebensbedrohlichen Schockreaktionen. Die wichtigsten klinischen Symptome der NMA innerhalb und außerhalb des Gastrointestinaltrakts sind in Tab. 4 zusammengefasst. Die NMA ist typischerweise eine Erkrankung der Haut, der Atemwege, des GI-Trakts oder einer Kombination hieraus. Von den Patienten mit echter NMA leidet etwa ein Drittel an gastrointestinalen Symptomen wie Übelkeit, Erbrechen, Krämpfe, Blähungen und Diarrhöen; andere klagen über Hautsymptome (Urtikaria, Quincke-Ödem, atopische Dermatitis), respiratorische Symptome (Rhinitis, Asthma bronchiale), Schocksymptomatik oder weniger gut definierte systemische Beschwerden (Migräne, Fatigue-Syndrom, Ödeme, Hypotension, Arthritis etc.). Letztere sind allerdings eher typisch für nicht immunologisch vermittelte Intoleranzen wie Histaminintoleranz $[29,30]$.

GI-Manifestationen. Während dermatologische, respiratorische und systemische Manifestationen allergischer Erkrankungen hinreichend bekannt und etabliert sind, gilt dies nicht für GI-Manifestationen, die häufig durch Nahrungsantigene ausgelöst werden und schwierig zu diagnostizieren und behandeln sind. Dies ist zurückzuführen auf unterschiedliche zugrunde liegende Mechanismen, die bislang nur ansatzweise geklärt sind, und auf limitierte diagnostische Methoden, um Betroffene auf objektiver Basis zu identifizieren. Diese Defizite sind z.T. dadurch begründet, dass der GI-Trakt weniger leicht zugänglich ist als z. B. die Haut, um neue Methoden zur Diagnostik und Therapie von NMA zu entwickeln [3].

Die NMA manifestiert sich an Haut, Atemwegen, GlTrakt oder einer Kombination hieraus. Etwa ein Drittel der NMA-Patienten leidet an GI-Symptomen wie Übelkeit, Erbrechen, Blähungen und Diarrhöen. Extraintestinale Symptome sind Migräne, Arthritis, generalisierte Ödeme, Hypotension und chronische Müdigkeit.

Anaphylaxie. Hervorzuheben ist, dass die NMA im Gegensatz zur NMI zu einer lebensbedrohlichen systemischen Anaphylaxie führen kann. Tatsächlich wird die NMA als Hauptursache für Anaphylaxie in Industrieländern wie USA und Europa angesehen [6]. Die Prävalenz der Erdnussallergie (0,5-7\% der Erwachsenen in den USA und UK) und ihre potenziell fatalen Konsequenzen haben bereits heute Auswirkungen auf Reglements in Schulkantinen bis hin zu den Fluggesellschaften. Fatale Anaphylaxie kann bereits durch geringste Allergenmengen ausgelöst werden wie beispielsweise solche, die durch einen Kuss übertragen werden. Gelegentlich tritt die Anaphylaxie nur unter gleichzeitiger

\section{Tabelle 4}

Klinische Symptome der Nahrungsmittelallergie.

\begin{tabular}{|c|c|c|}
\hline Organ & Krankheitsbild & Betroffene \\
\hline GI-Trakt & $\begin{array}{l}\text { orales Allergiesyndrom (OAS) } \\
\text { eosinophile Entzündungen } \\
\text { IgE-unabhängige Entzündungen } \\
\text { Zöliakie } \\
\text { Reizdarmsyndrom? }\end{array}$ & $\begin{array}{l}\text { alle } \\
\text { alle } \\
\text { Kinder } \\
\text { alle } \\
\text { Erwachsene }\end{array}$ \\
\hline Atemwege & $\begin{array}{l}\text { Heuschnupfen, Asthma } \\
\text { Otitis serosa }\end{array}$ & $\begin{array}{l}\text { alle } \\
\text { Kinder }\end{array}$ \\
\hline Haut & $\begin{array}{l}\text { Urtikaria } \\
\text { Neurodermitis }\end{array}$ & $\begin{array}{l}\text { alle } \\
\text { Kinder }\end{array}$ \\
\hline Gelenke & Arthritis etc. & Erwachsene \\
\hline Nervensystem & $\begin{array}{l}\text { Migräne, Kopfschmerzen } \\
\text { anhaltende Müdigkeit } \\
\text { psychische Auffälligkeiten } \\
\text { hyperkinetisches Syndrom }\end{array}$ & $\begin{array}{l}\text { Erwachsene } \\
\text { Erwachsene } \\
\text { Erwachsene } \\
\text { Kinder }\end{array}$ \\
\hline Herz-Kreislauf-System & $\begin{array}{l}\text { Gefäßentzündungen } \\
\text { Ödeme } \\
\text { anaphylaktischer Schock }\end{array}$ & $\begin{array}{l}\text { alle } \\
\text { alle } \\
\text { alle }\end{array}$ \\
\hline
\end{tabular}


körperlicher Anstrengung auf. Beispielsweise kann durch Getreide ausgelöste, anstrengungsinduzierte Anaphylaxie durch das Major-Allergen, Omega-5-Gliadin (Tri a 19) getriggert werden, nachdem die Anstrengung eine Aktivierung von Gewebstransglutaminase in der Darmmukosa bewirkt hat, die zu einer Kreuzvernetzung von Omega-5-Gliadinpeptiden führt, wodurch größere Allergenkomplexe gebildet werden, die dann eine anaphylaktische Reaktion auslösen können. Azetylsalizylate und andere NSAR können ebenfalls zu einer Verstärkung allergischer Symptome beitragen.

NMA kann im Gegensatz zur NMI zu einer lebensbedrohlichen systemischen Anaphylaxie führen.

\section{Organmanifestationen Haut, Lunge, Darm}

Haut. Hautmanifestationen schließen die atopische Dermatitis (häufig assoziiert mit gesteigerter Darmpermeabilität) und die Urtikaria (besonders in Assoziation mit anstrengungsinduzierten Symptomen) ein [31,32]. Dermatitis herpetiformis tritt bei glutensensitiver Enteropathie auf, die Hautläsionen werden effektiv allein durch glutenfreie Diät behandelt.

Respirationstrakt. Respiratorische Manifestationen der NMA umfassen die bronchiale Hyperreaktivität, das Asthma bronchiale, die allergische Rhinitis und möglicherweise auch die seröse Otitis media. Wenngleich Asthma vorwiegend mit inhalativen Allergenen assoziiert wird, muss bedacht werden, dass NMA ein relevantes Risiko für lebensbedrohliche Asthmareaktionen im Kindesalter darstellt [33].

GI-Trakt. Gastrointestinale Manifestationen der NMA sind bei Kindern typischerweise die nahrungsproteininduzierten Proktitiden bzw. Enteropathien, evtl. kombiniert mit atopischer Dermatitis [2]. In neuerer Zeit wurden zusätzlich die eosinophile Ösophagitis und die allergische Obstipation als Manifestationen der NMA beschrieben [21,34]. Bei Teenagern und Erwachsenen ist das orale Allergiesyndrom (OAS) häufigste Manifestationsform einer NMA, wenngleich auch andere Manifestationen wie eosinophile Enteropathien und die Zöliakie vorkommen [3].

\section{- Orales Allergiesyndrom (OAS)}

Das OAS wird typischerweise durch pflanzliche Proteine ausgelöst, die mit bestimmten inhalativen Antigenen Kreuzreaktionen aufweisen, insbesondere mit Birken-, Ragweed- (beifußblättriges Traubenkraut, Ambrosia) und Mugwort-Pollen (Beifuß). Exposition mit kreuzreagierenden Nahrungsmitteln kann zu Pruritus, Kribbeln und Schwellung der Zunge, der Lippen, des Gaumens oder des Pharynx und gelegentlich auch zu Bronchospasmus oder sogar systemischen Reaktionen führen, die üblicherweise bereits wenige Minuten nach Allergeningestion auftreten. Da diese Reaktionen fast ausschließlich IgE-vermittelt sind, kann die Diagnose in der Regel mittels Pricktest oder Messung von spezifischem IgE im Blut bestätigt werden.

\section{- Intestinales Allergiesyndrom (IAS)}

Typischerweise treten die gastrointestinalen Symptome (Übelkeit, Erbrechen, abdominelle Schmerzen und Diarrhö) in Verbindung mit extraintestinalen Symptomen auf. Die typischen Allergene, die solche Reaktionen auslösen können, sind Milch, Ei, Erdnuss, Fisch und Meeresfrüchte, je nachdem welche Essgewohnheiten dominieren [3].

\section{- Latex-Nahrungsmittelallergie-Syndrom}

Das Latex-Nahrungsmittelallergie-Syndrom, auch Latex-Früchte-Syndrom genannt, ist eine besondere Form der NMA mit steigender Prävalenz, wobei die Latexallergie in 21 - 58\% der Fälle gemeinsam mit NMA auftritt [35]. Weltweit sind Banane, Avocado, Walnuss und Kiwi die häufigsten Auslöser von nahrungsmittelassoziierten Symptomen bei Latexallergie. Diese Nahrungsmittel können bei Latexallergikern dieselben Symptome wie Latex induzieren, z. B. Pruritus, Ekzem, OAS, Asthma, GI-Beschwerden und generalisierte Anaphylaxie.

\section{- Nahrungsinduzierte Enteropathie und Enterokolitis/Proktitis}

Die nahrungsinduzierte Enteropathie ist eine Erkrankung des Kindesalters und ist gekennzeichnet durch protrahierte Diarrhöen und Erbrechen, was zu dem klinischen Bild einer Malassimilation führt. Eiweißverlust-Enteropathie kann zu Ödemen, abdomineller Distension, Nausea, Erbrechen, Diarrhö und Anämie führen. Differenzialdiagnostisch sind infektiöse und metabolische Erkrankungen, Lymphangiektasien und die Zöliakie zu berücksichtigen. Zugrunde liegende Mechanismen umfassen Immunkomplexbildungen und abnorme T-Zell-Reaktionen nach Genuss von Milch, Soja und anderen Nahrungsmitteln wie Ei, Fisch, Getreide, Reis, Gemüse und Fleisch [2]. Normalerweise ist dabei kein spezifisches IgE gegen diese Nahrungsmittel nachweisbar. Die Diagnose basiert auf endoskopischen und histologischen Befunden (vermehrt intraepitheliale Lymphozyten und eosinophile Granu- 
lozyten, Villusatrophie) sowie auf Eliminationsdiät und Reexposition.

\section{Zöliakie}

Diese früher auch einheimische Sprue genannte Erkrankung betrifft ca. $1 \%$ der Bevölkerung, was frühere Schätzungen zur Häufigkeit bei Weitem übertrifft. Die orale Aufnahme von Gliadin, das in Getreide und Reishäutchen enthalten ist, induziert eine Enteropathie bei genetisch vorbelasteten Individuen. Durch Eliminationsdiät kann eine Normalisierung der Darmanatomie und -funktion erreicht und die Symptomatik (Diarrhö, Gewichtsverlust, aber auch Müdigkeit, Abgeschlagenheit und Dyspepsie) reduziert bzw. aufgehoben werden. Gluten muss üblicherweise lebenslang eliminiert werden [36].

Die häufigsten Manifestationen von NMA im Kindesalter sind Diarrhöen und atopische Dermatitis, im Erwachsenenalter das orale Allergiesyndrom, das meist durch mit Pollen kreuzreagierende Nahrungsmittel ausgelöst wird.

\section{Eosinophile Ösophagitis und gastro- ösophageale Refluxkrankheit (GERD)}

Untersuchungen zur Milchelimination bei Kindern mit Refluxerkrankung zeigten, dass ca. ein Drittel der Refluxerkrankungen durch Kuhmilch verursacht wird [2]. In solchen Fällen führt eine klassische medikamentöse Antirefluxtherapie zu keiner Besserung und die histologische Untersuchung zeigt eine ausgeprägte Infiltration mit eosinophilen Granulozyten, was der Erkrankung den Namen gab. Typische Symptome umfassen Erbrechen, retrosternale Schmerzen und Dysphagie, bis hin zur Verlegung der Speiseröhre mit Nahrung aufgrund von ösophagealen Strikturen. Nahrungsmittelallergien finden sich assoziiert bei den meisten Betroffenen, die nicht selten zusätzlich an Asthmasymptomen leiden. Neuere Studien zeigten, dass diese Erkrankung keineswegs auf Kinder beschränkt ist, sondern auch Erwachsene in einem noch weitgehend unbekannten Ausmaß betreffen kann [21, 34].

\section{Eosinophile Gastroenterokolitis}

Die eosinophile Gastroenterokolitis ist eine seltene heterogene Erkrankung mit eosinophiler Infiltration der Magen-/Darmmukosa. Die Lokalisation und die Tiefe der Infiltration mit eosinophilen Granulozyten bestimmen die unterschiedlichen Manifestationsformen und sind Grundlage für die Klassifikation in mukosale, muskuläre und serosale Formen der Erkrankung. Abdominelle Schmerzen, Erbrechen und Diarrhö kommen bei über $50 \%$ der Patienten gleichzeitig vor. In mehr als zwei Dritteln der Fälle ist auch eine Eosinophilie im peripheren Blut zu sehen. Die Differenzialdiagnose der eosinophilen Gastroenterokolitis umfasst bei Kindern Parasitosen, CED, Bindegewebserkrankungen, Tumorerkrankungen und Medikamentenallergien. Die eosinophile Gastroenterokolitis selbst ist eng assoziiert mit Nahrungsmittelallergie und anderen atopischen Erkrankungen bzw. einer Familienanamnese mit Allergien in 50-70\% der Fälle. Die Diagnose basiert auf dem histologischen Nachweis einer Gewebseosinophilie, oft mit milder Mastozytose. [21, 34].

Weniger lang bekannte Manifestationsformen von Nahrungsmittelallergien sind eosinophile Erkrankungen des Gastrointestinaltrakts (eosinophile Ösophagitis, eosinophile Gastroenterokolitis), die aber auch eine nicht allergische Genese haben können.

\section{Klinik der Nahrungsmittelintoleranzen}

Die Mehrzahl der abnormalen Reaktionen auf Nahrungsmittel ist nicht immunologischer Genese, aber aufgrund ihrer Häufigkeit von besonderer Relevanz. Diese Nahrungsmittelintoleranzen umfassen beispielsweise bakterielle Lebensmittelvergiftungen, postinfektiöse Reizdarmsymptomatik und chronische Intoleranzreaktionen, die hier näher besprochen werden sollen (Tab.2).

\section{- Pseudoallergische und pharmakologische Reaktionen}

Solche Reaktionen werden durch Nahrungsmittel induziert, die die IgE-abhängige Mastzelldegranulation imitieren, indem sie die Mastzellen IgE-unabhängig aktivieren. Trigger sind meist Nahrungsmittelzusatzstoffe wie Sulfide, Tartrazin und Glutamat, die Darmsymptome auslösen, die nicht selten mit Asthma vergesellschaftet sind. Glutamat verursacht bei Betroffenen innerhalb weniger Minuten charakteristische Symptome wie Hitzewallungen, Engegefühl, Kopfschmerzen und epigastrische Beschwerden („Chinarestaurant-Syndrom“, weil Glutamat in der chinesischen Küche reichlich als Gewürz bzw. Geschmacksverstärker Einsatz findet). Wie bei der NMA ist Eliminationsdiät die Therapie der Wahl-vorausgesetzt, das auslösende Nahrungsmittel konnte identifiziert werden. Pharmakologische Reaktionen auf Nahrungsmittel oder Zusatzstoffe sind ebenfalls häufige Formen einer Nahrungsmittelunverträglichkeit, wenngleich die Symptome meist extragastrointestinal lokalisiert sind. 


\section{- Histaminintoleranz}

Biogene Amine wie Histamin, Serotonin oder Tyramin können nahrungsmittelallergieähnliche Symptome auslösen wie Kopfschmerzen, Hypotension, Erytheme und GI-Symptome. Die Pathophysiologie der Histaminintoleranz umfasst eine durch einen gestörten Histaminabbau bedingte Akkumulation von Histamin und somit eine erhöhte Empfindlichkeit gegen eher kleine Mengen von Histamin in Nahrungsmitteln. Ursache ist ein Mangel an Diaminooxidase (DAO), dem wichtigsten Abbauenzym für Histamin, oder an DAO-Koenzymen wie Vitamin $B_{6}$ und evtl. Vitamin C. Die Bestimmung von Plasmahistamin und DAO sowie Provokationstests mit Histamin erlauben die Sicherung der Diagnose $[29,30]$.

\section{- Laktoseintoleranz}

Diese Erkrankung, auch als Laktosemalassimilation bezeichnet, repräsentiert die häufigste Form einer NMI und wird meist durch eine abnehmende Expression von Laktase im Darm mit zunehmendem Lebensalter bedingt, kann in seltenen Fällen aber auch primär genetisch bedingt sein. Die Symptome wie Blähungen, Flatulenz und Diarrhö treten üblicherweise dosisabhängig auf. Ein sekundärer Laktasemangel kann bei viraler Gastroenteritis, Morbus Crohn und Zöliakie auftreten. Für die Behandlung der Patienten ist es wichtig zu wissen, dass Betroffene im Gegensatz zu Milchallergikern

- nicht an einer potenziell lebensbedrohlichen Erkrankung leiden und

- Milchprodukte mit relativ wenig Laktose (durch Zusatz laktaseproduzierender Bakterien) wie Käse und Joghurts vielfach vertragen [37].

\section{- Psychologische Intoleranz}

Bei einzelnen Personen wurde eine psychologische Ursache für die Nahrungsmittelunverträglichkeit vermutet [38]. Dabei handelt es sich um eine besonders schwierige Form einer NMI, denn die Mechanismen sind kaum bekannt. Viele dieser Patienten leiden häufiger an Hypochondrie, Hysterie und Somatisierungen, wobei die NMI typischerweise in Provokationstests nicht bestätigt werden kann. Mögliche Ursachen solcher Störungen sind negative Erfahrungen mit bestimmten Nahrungsmitteln, z. B. schwere Vergiftungen oder Essenszwang in der Kindheit. Konditionierungsmechanismen könnten ebenfalls beteiligt sein [24].

\section{- Physiologische Nahrungsmittelintoleranz}

Hierbei handelt es sich womöglich um die häufigste Form einer NMI auf bestimmte Nahrungsbestandteile oder -zusatzstoffe. Beispielsweise kann Stärke aus
Gemüse oder Getreide zu Gasproduktion im Kolon führen. Andere Nahrungsstoffe sind bekannt dafür, dass sie den Tonus des unteren Ösophagussphinkters reduzieren bzw. zu einer verzögerten Magenentleerung führen, was in Dyspepsie resultiert. Solche physiologischen Reaktionen auf Nahrungsmittel werden besonders von Patienten mit RDS berichtet. Die Untersuchung auf NMI ist in dieser Gruppe von Patienten deshalb von Bedeutung, weil gezeigt werden konnte, dass eine gezielte Eliminationsdiät tatsächlich zu einer Besserung des Beschwerdebildes führt [39].

Abnormale Reaktionen auf Nahrungsmittel sind mehrheitlich nicht immunologischer Genese und äußern sich charakteristischerweise mit Hitzewallungen, Engegefühl, Kopfschmerzen und Hypotension, Erythemen, aber auch GI-Beschwerden (epigastrische Beschwerden, Blähungen, Flatulenz und Diarrhö).

\section{Diagnostik}

Kürzlich sind neue Leitlinien zur Diagnostik und Therapie von Nahrungsmittelallergien aus den USA [40, 41], Großbritannien [42] und Deutschland [43,44] veröffentlicht worden. Darüber hinaus finden sich Leitlinien und Positionspapiere der Deutschen Gesellschaft für Allergologie und klinische Immunologie (DGAKI) zum Thema unter http://dgaki.de/leitlinien/aktuelleleitlinien/.

\section{Grundlagen: Anamnese und Ausschlussdiagnostik}

Grundlage der Diagnostik ist eine sorgfältig erhobene Anamnese zu den Nahrungsmitteln, die nicht vertragen werden und die mit spezifischen Symptomen korrelieren. Offene Provokationstests sind hilfreich, wenngleich sie subjektiver Natur sind und einer Bestätigung durch objektivere Testmethoden bedürfen, bevor eine dauerhafte Eliminationsdiät empfohlen werden kann. Aufgrund der Tatsache, dass kein Test die Verdachtsdiagnose NMA eindeutig bestätigen kann, ist eine sorgfältige Ausschlussdiagnostik erforderlich, die bei Erwachsenen umfangreicher sein muss als bei Kindern (Tab.5). Die Allergietests, die im Folgenden besprochen werden sollen, dienen entweder dem Nachweis der Unverträglichkeit bestimmter Nahrungsmittel oder des immunologischen Mechanismus der Unverträglichkeit. Zur vollständigen Diagnose sollten beide Nachweise erbracht sein (Tab.6). 


\begin{tabular}{ll}
\hline Tabelle $\mathbf{5}$ & \\
\hline Ausschlussdiagnostik. & \\
\hline Erwachsene & Kinder \\
\hline - Nahrungsintoleranzen nicht immuno- & - Infektionen \\
logischer Genese (z. B. LTT) & - Sprue \\
- Reflux, Ulkus, Gastritis & \\
- Sprue, Morbus Whipple, CED, mikro- & \\
& skopische Kolitis \\
- Infektionen & \\
- Tumoren
\end{tabular}

LTT: Lymphozytentransformationstest.

Standardisiertes Vorgehen. Obwohl alle Allergietests aufgrund begrenzter Sensitivität und Spezifität weder die Verdachtsdiagnose bestätigen noch ausschließen können, erlangen sie gute Vorhersagewerte, wenn sie bei einem sorgfältig vorselektierten Patientenkollektiv angewandt werden. Dies bedeutet, dass Patienten mit Verdacht auf NMA nach standardisierten Schritten diagnostiziert werden sollten [40-44]. Bei Verdacht auf NMA mit gastrointestinaler Manifestation hat sich in der klinischen Praxis ein standardisiertes Flussschema bewährt (Abb.3).

Die Diagnostik von NMA und NMI sollte so weit wie möglich leitlinienorientiert erfolgen. Ausschlussdiagnostik ist obligatorischer Beststandteil der Abklärung von NMA und NMI.

\section{Allergie-Hauttests}

Allergie-Hauttests, auch Pricktests genannt, sind einfach durchzuführende, kostengünstige Testmethoden zur Evaluation von Sensibilisierungen bei Kindern und Erwachsenen [43]. Allerdings sind Limitationen zu bedenken, die sich insbesondere auf einen begrenzten positiven Vorhersagewert dieses Testverfahrens begründen. Eine weitere Limitation des Pricktests ist bedingt durch die ungenügende Standardisierung und Stabilität vieler Extrakte von Nahrungsmittelallergenen, die zur Testung verwendet werden. Dieses Problem könnte reduziert werden durch die Verwendung rekombinanter Allergene, die in zunehmender Anzahl zur Verfügung stehen, bzw. durch die Verwendung von nativen Nahrungsmitteln mittels Prick-to-Prick-Test, wobei zuerst das Nahrungsmittel, dann die Haut des Patienten mit derselben Lanzette „geprickt“ werden. Neuerdings steht auch der Epikutantest zur kutanen

\section{Tabelle 6}

\begin{tabular}{|c|c|}
\hline Nachweis der Unverträglichkeit & $\begin{array}{l}\text { Nachweis des immunologischen } \\
\text { Mechanismus }\end{array}$ \\
\hline - Anamnese & - Hauttests, RAST \\
\hline $\begin{array}{l}\text { - offene Provokation, Diätetik } \\
\text { - kontrollierte Provokation (oral: }\end{array}$ & $\begin{array}{l}\text { - Messung von Entzündungsmediato- } \\
\text { ren ( } \pm \text { kontrollierte Provokation) }\end{array}$ \\
\hline $\begin{array}{l}\text { DBPCFC/lokal: COLAP) } \\
\text { - Release-Tests (BHRT, CAST, TABOX etc.) }\end{array}$ & $\begin{array}{l}\text { - Visualisierung von Mastzelldegranu- } \\
\text { lation ( } \pm \text { kontrollierte Provokation) }\end{array}$ \\
\hline
\end{tabular}

DBPCFC: double-blind placebo-controlled food challenge, COLAP: colonoscopic allergen provocation, BHRT: basophil histamin-releasing test, CAST: chemiluminicence allergo-sorbent test, TABOX: Provokationstest an Schleimhautbiopsien, RAST: radio-allergo-sorbent-test.

Testung verzögerter Reaktionen zur Verfügung, der derzeit in klinischen Studien evaluiert wird.

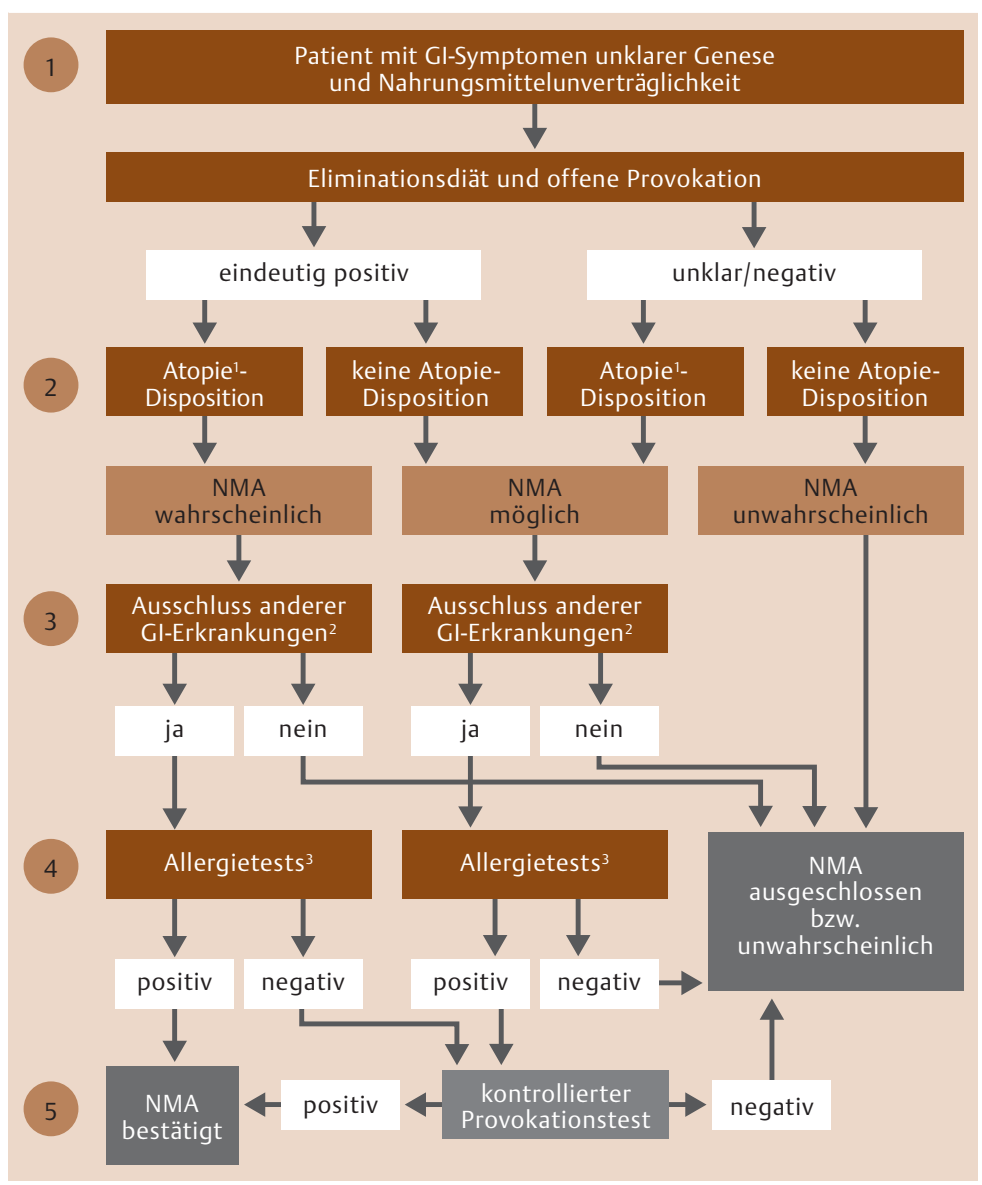

Abb.3 Algorithmus zur Diagnostik von Nahrungsmittelallergien (Quelle: [4], modifiziert). 1: definiert als Vorhandensein anderer Allergien als NMA beim Patienten oder Verwandten ersten Grades oder positive Allergietests in der Vergangenheit; ${ }^{2}$ : s. Tab. 5, ${ }^{3}$ : s. Tab. 6. 


\section{In-vitro-Allergiediagnostik}

Spezifisches IgE im Serum. Eine Alternative bzw. Ergänzung der Hauttests ist die Messung von spezifischem IgE im Serum, ein Laborverfahren, das eine höhere Spezifität und aufgrund der Unabhängigkeit vom Untersucher eine höhere Reliabilität aufweist. Dieser Test, der auch unter dem Begriff RAST (RadioAllergo-Sorbent-Test, weil spezifisches IgE früher radioaktiv gemessen wurde) bekannt ist, kann auch bei Patienten mit Hautbeteiligung, z. B. atopischer Dermatitis, durchgeführt werden, wo Hauttests nicht empfohlen werden. Eine mögliche Ursache für die häufig berichtete Diskrepanz zwischen Ergebnissen aus Anamnese, Hauttests und Messung von spezifischem IgE liegt darin, dass NMA im GI-Trakt durch lokal produziertes IgE vermittelt wird, was sich nicht in veränderten IgE-Serumspiegeln niederschlagen muss.

IgE-unabhängige Parameter. Schließlich wird auch die Messung IgE-unabhängiger Parameter empfohlen, beispielsweise von Eosinophilen-Mediatoren wie ECP und EPX im Serum und in den Fäzes, weil damit auch IgEunabhängige allergische Reaktionen erfasst werden können [3,22].

Allergietests (Hauttests: Prick u. Ä.; Bluttests: spezifisches $(\mathrm{gE}$ ) können in der Regel aufgrund begrenzter Sensitivität und Spezifität die Verdachtsdiagnose gastrointestinale NMA weder bestätigen noch ausschließen. Daher beruht die Diagnose wesentlich auf einer negativen Ausschlussdiagnostik, vor allem für infektiöse, chronisch entzündliche und maligne Magen-DarmErkrankungen.

\section{Allergen-Provokationsverfahren}

Orale Provokation. In unklaren Fällen ist ein kontrollierter Provokationstest, in der Regel in Form einer „double-blind placebo-controlled food challenge“ (DBPCFC), erforderlich, wobei Nahrungsantigene oral oder direkt intestinal über eine Magensonde bzw. in Form einer Gelatinekapsel, die das Allergen enthält, zugeführt werden. Das Verfahren der DBPCFC wird als Goldstandard für die Bestätigung der Diagnose NMA angesehen und sollte in allen unklaren Fällen obligater Bestandteil in der Diagnostik sein $[40,42,44]$. Andererseits weist auch dieses Verfahren Schwächen auf, insbesondere, wenn es um die Abklärung einer NMA mit gastrointestinaler Manifestation geht:
- Das Read-out dieses Tests ist hinsichtlich gastrointestinaler Symptome kaum standardisiert und validiert, d. h. es handelt sich eher um einen subjektiven als einen objektiven Test.

- Es wird keine immunologische Reaktion verifiziert, d.h. der Test prüft Nahrungsmittelunverträglichkeit, aber nicht -allergie [3].

Lokale Testung. Einige Versuche wurden unternommen, ein gastrointestinales Äquivalent des AllergieHauttests zu entwickeln, indem Nahrungsallergene in die Magen- oder Darmmukosa appliziert und Reaktionen wie Schleimhautrötung oder -schwellung erfasst werden. Dieser Ansatz wurde bereits in den 30er Jahren konzipiert und danach in Form von gastralen, duodenalen und zuletzt kolonischen Provokationen weiterentwickelt [23].

\section{Diagnostik von Nahrungsmittel- intoleranzen}

Zur Diagnostik von NMI stehen wenige objektive, validierte Testverfahren zur Verfügung. Insofern sind Anamnese, Eliminationsdiäten und insbesondere Provokationsverfahren hier von zentraler Bedeutung.

Kohlenhydratmalabsorption. Hervorzuheben sind die Atemtestverfahren, die nach Provokation für die Diagnose von Kohlenhydratmalabsorption (z.B. Laktose, Fruktose) verwendet werden. Einzelheiten dazu sind an anderer Stelle beschrieben $[45,46]$. Darüber hinaus gibt es die Möglichkeit, Enzymaktivitäten und Polymorphismen für enzymkodierende Gene zu messen. Für die Laktosemalabsorption kann die Enzymaktivität in der Darmmukosa bestimmt werden (nicht als Routineverfahren etabliert), außerdem sind Polymorphismen für das Laktasegen beschrieben, die mit der Wahrscheinlichkeit korrelieren, im Verlauf des Lebens an Laktosemalabsorption zu erkranken. Allerdings können diese Tests, die die aktuelle Krankheitsaktivität nicht spiegeln, in keiner Weise den Laktose- $\mathrm{H}_{2}$-Atemtest ersetzen.

Histaminintoleranz. Für die Diagnose der Histaminintoleranz kann das histaminabbauende Enzym Diaminooxidase (DAO) bzw. Kofaktoren der DAO wie Vitamin $B_{6}$ bestimmt werden, Laborwerte, die in Kombination mit Anamnese und Provokationsverfahren die Sicherung der Diagnose ggf. unterstützen [29]. Allerdings wird die In-vitro-Diagnostik der Histaminintoleranz mittels DAO, Histamin im Plasma und Methylhistamin im Urin zunehmend kritisch bewertet, weil die 
Validierungsdaten für solche Verfahren unzureichend sind [30].

Für die Diagnostik von NMI gibt es nur wenige objektive, validierte Testverfahren, weshalb Anamnese, Eliminations- und Provokationsverfahren hier von zentraler Bedeutung sind.

\section{Therapie}

\section{Nahrungsmittelintoleranzen}

Da die Mechanismen der Nahrungsmittelintoleranzen unbekannt bzw. pharmakologischen Interventionen nicht zugänglich sind, steht als Therapiestrategie bei Nahrungsmittelintoleranzen lediglich die Eliminationsdiät zur Verfügung. Diese erfordert eine eingehende, individuelle Beratung und Schulung des Patienten. Er muss lernen, seine Ernährung weg von Fertignahrungsmitteln auf Primärnahrungsmittel umzustellen und Etiketten zu lesen.

\section{Nahrungsmittelallergien}

\section{- Eliminationsdiät}

Die Grundlage für die Behandlung einer Nahrungsmittelallergie ist ebenfalls die Vermeidung der Exposition mit dem/den symptomauslösenden Allergen(en). Dies ist bei Allergien wie Erdnussallergie von besonderer Relevanz, bei denen bereits winzige Allergenspuren relevante Reaktionen auslösen können. Die Praktikabilität solcher Eliminationsdiäten ist jedoch begrenzt. Sie erfordert eine geschulte Beratungskraft, Zeit und hohe Motivation von Seiten des Betroffenen. Nahrungsmittelallergiker sollten auch lernen, Etiketten auf Nahrungsmitteln zu lesen und hinsichtlich versteckter bzw. kreuzreagierender Allergene zu verstehen [40-42].

Eliminationsdiät ist die Grundlage der Therapie von NMI und NMA. Eine geschulte Beratungskraft, Zeit und große Motivation seitens des Betroffenen sind erforderlich.

\section{- Supplementäre medikamentöse Therapie}

Wenn eine Eliminationsdiät nicht vollständig durchgeführt werden kann oder wenn nicht alle auslösenden Nahrungsmittel klar identifiziert werden können, ist eine ergänzende medikamentöse Therapie erforderlich. Hierfür ist eine magen-/darmtaugliche Präparation von Cromoglicinsäure verfügbar [47]. In schwereren Fällen kann eine (passagere) Therapie mit Korti- kosteroiden unvermeidlich sein. Inwieweit lokal wirksame Steroide wie Budesonid für die Therapie der gastrointestinalen Nahrungsmittelallergie geeignet sind, wurde in kontrollierten Studien bislang nicht getestet [3]. Bislang gibt es keine eindeutige Datenlage für den Einsatz von oraler oder systemischer Desensibilisierung, für prophylaktische medikamentöse Therapie oder ähnliche Ansätze zur Behandlung von NMA.

Als supplementäre medikamentöse Therapie von NMA kommen Cromoglicinsäure und (lokal wirksame) Kortikosteroide in Frage. Für den Einsatz von oraler oder systemischer Desensibilisierung bei NMA gibt es keine eindeutige Datenlage.

\section{Medikamentöse Notfalltherapie}

Da eine ungewollte Exposition mit Nahrungsantigenen nicht immer vermieden werden kann, müssen Patienten mit Anaphylaxie-Anamnese mit einem sog. Notfallset an Medikamenten ausgerüstet werden. Zentraler Bestandteil des Sets ist Adrenalin, in dessen Anwendung in Notfallsituationen Betroffene sorgfältig eingewiesen werden müssen. Weiterhin sollte das Set ein Kortikosteroid ( $2 \times 100 \mathrm{mg}$ Prednisolon-Äquivalent) sowie ein Antihistaminikum (z.B. $2 \times 2$ mg Tavegil) enthalten [48].

Patienten mit NMA und Anaphylaxie-Anamnese müssen mit einem sog. Notfallset an Medikamenten (Adrenalin, Steroid, Antihistaminikum) ausgerüstet werden.

\section{- Prävention}

Hypoallergene Kostformen werden während der Schwangerschaft bzw. der Stillzeit empfohlen für atopische Mütter, um die Inzidenz der Nahrungsmittelallergie bei ihren Kindern zu reduzieren. Nahrungsmittel mit besonders hohem Allergiepotenzial sollten verzögert in den Speiseplan der Risiko-Babys eingeführt werden, um die Wahrscheinlichkeit einer NMA zu minimieren.

Eine verbindliche Empfehlung zur Verwendung von Probiotika zur Prävention von Nahrungsmittelallergien kann nicht gegeben werden, da entsprechende Studien unterschiedliche Resultate erbrachten (s. „Studienergebnisse-Probiotika“).

Hypoallergene Produkte sind solche, bei denen Milcheiweiß mittels Hitze oder Enzymen teilweise bzw. nahezu vollständig hydrolysiert wurde. Eine eindeutige Therapieempfehlung für solche Produkte besteht nicht. 


\section{Studienergebnisse}

\section{Probiotika}

Anfang 2000 wurden Studien publiziert, nach denen Probiotika, wie z. B. Lactobacillus rhamnosus GG, in der Lage sind, die Allergieinzidenz bei Kindern aus Hochrisikofamilien für die Entwicklung allergischer Erkrankungen zu reduzieren. Beispielsweise wird die Prävalenz der nahrungsinduzierten atopischen Dermatitis durch die Behandlung mit Lactobacillus GG während und unmittelbar nach der Schwangerschaft um ca. $50 \%$ reduziert, wie Erhebungen 2 bzw. 4 Jahre nach Geburt der Kinder belegten [49]. Neuere Studien haben diese positiven Ergebnisse jedoch in Frage gestellt [50-52]. Deshalb kann derzeit keine verbindliche Empfehlung zur Verwendung von Probiotika zur Prävention von Nahrungsmittelallergien ausgesprochen werden.

\section{- Ausblick: Neue Therapieansätze}

Ein spannendes Gebiet der modernen Allergieforschung ist die Entwicklung neuer therapeutischer Ansätze wie tolerogene Peptide, rekombinante Epitope zur Hyposensibilisierung, Anti-IgE-Antikörper und DNA-Impfung mit Allergen-DNA, Anti-Zytokin-Antikörper bzw. Zytokinrezeptor-Antagonisten gegen Th2-Zytokine wie IL-4 sowie Anti-c-kit-Antikörper als neue Antimastzellenmedikamente [4].
Modifikation von Antigenstrukturen. Darüber hinaus sind Methoden zur genetischen bzw. chemischen Modifikation von Antigenstrukturen von Nahrungsmittelallergenen mit dem Ziel der Reduzierung des allergenen Potenzials entwickelt worden. Beispielsweise bewirkt der Austausch einer einzigen Aminosäure im Bereich der Proteinsequenz der IgE-Bindungsstelle des Erdnussallergens den kompletten Verlust der IgE-Bindung dieses Allergens. Valenta und Mitarbeiter konnten auf molekularer Ebene zeigen, dass solche Methoden auf nahezu alle klonierten Allergene anwendbar sind, was vollkommen neue Ansätze zur Impfung gegen TypI-Allergien bietet [28].

Anti-IgE-Therapie. Antikörper gegen den Fc-Teil des IgE, das an den hochaffinen IgE-Rezeptor bindet, konnten in tierexperimentellen und klinischen Studien mit Asthmatikern erfolgreich eingesetzt werden und bieten auch ein Potenzial zur Therapie von NMA. Tatsächlich wurde die Anti-IgE-Therapie bei Patienten mit Erdnussallergie bereits erfolgreich eingesetzt [53].

\section{Kernaussagen}

\section{Epidemiologie}

- Nahrungsmittelunverträglichkeiten betreffen ca. 20-30\% der Allgemeinbevölkerung in westlichen Ländern.

- Bei etwa einem Viertel der betroffenen Kinder und einem Zehntel der betroffenen Erwachsenen liegt der Unverträglichkeit eine Allergie, d. h. eine immunologisch vermittelte Unverträglichkeitsreaktion zugrunde. In einem Drittel dieser Fälle treten gastrointestinale Symptome auf.

\section{Pathophysiologie und klinisches Bild}

- Nahrungsmittelallergien (NMA) werden durch IgE-abhängige oder IgE-unabhängige immunologische Reaktionen ausgelöst, die zu einer Entzündungsreak- tion führen, an der Mastzellen, eosinophile Granulozyten und andere Zellen beteiligt sind. Sowohl genetische als auch Umweltfaktoren werden ursächlich diskutiert.

- Die NMA ist typischerweise eine Erkrankung der Haut, der Atemwege, des GITrakts oder einer Kombination hieraus.

- Die NMA kann im Gegensatz zu Intoleranzen zu einer lebensbedrohlichen systemischen Anaphylaxie führen.

\section{Diagnostik und Therapie}

- Neue Erkenntnisse zur Wechselwirkung zwischen angeborenem Immunsystem und intestinaler Mikrobiota haben innovative pathophysiologische Konzepte generiert, die epidemiologische Beob- achtungen unterstützen, nach denen ländlicher Lebensstil protektiv wirkt gegen allergische und immunologische Erkrankungen.

- Die Entwicklung rekombinanter Allergene und Allergenvarianten hat die Möglichkeiten der Diagnostik verbessert und bietet neue Therapieoptionen an, wie beispielsweise die Hyposensibilisierung und die Induktion von immunologischer Toleranz.

- Nahrungsmittelintoleranzen, das sind nicht immunologisch vermittelte Nahrungsmittelunverträglichkeiten, die vielfach durch spezifische Enzymdefizienzen verursacht werden, sind differenzialdiagnostisch von Nahrungsmittelallergien abzugrenzen. 


\section{Über den Autor}

\section{Stephan C. Bischoff}

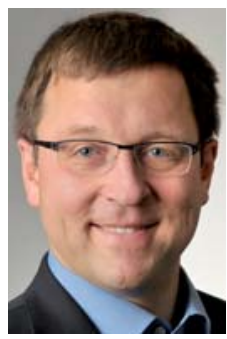

Studium der Medizin in Mainz und Straßburg. 1989 wissenschaftlicher Assistent im Institut für Klinische Immunologie, Universitätsklinik Bern/Schweiz. Ab 1992 Arzt und später Oberarzt in der Abt. Gastroenterologie der Medizinischen Hochschule Hannover. Dort Habilitation und Erwerb der Facharztkunde für Innere Medizin, Allergologie, Gastroenterologie und Ernährungsmedizin. 2002-2003 Gastprofessur an der Columbia Universität New York. 2004 Ernennung zum Ordentlichen Professor für Ernährungsmedizin und Prävention und Geschäftsführender Direktor des Instituts für Ernährungsmedizin an der Universität Hohenheim in Stuttgart. Seit 2009 Ärztlicher Direktor des Zentrums für Ernährungsmedizin der Universitäten Hohenheim und Tübingen. Forschungsschwerpunkte: Adipositas und adipositasassoziierte Erkrankungen, Malnutrition und Nahrungsmittelunverträglichkeiten sowie mukosale Immunologie, Probiotika und Darmbakterien.

\section{Korrespondenzadresse}

Univ.-Prof. Dr. med. Stephan C. Bischoff

Institut für Ernährungsmedizin

Universität Hohenheim

Fruwirthstraße 12

70593 Stuttgart

E-Mail: bischoff.stephan@uni-hohenheim.de

\section{Literatur}

1 Coombs RR. The Jack Pepys Lecture. The hypersensitivity reactions - some personal reflections. Clin Exp Allergy 1992; 22: $673-680$

2 Sicherer $\mathrm{SH}$. Clinical aspects of gastrointestinal food allergy in childhood. Pediatrics 2003; 111: 1609-1616

3 Bischoff S, Crowe SE. Gastrointestinal food allergy: new insights into pathophysiology and clinical perspectives. Gastroenterology 2005; 128: 1089-1113

4 Iacono G, Cavataio F, Montalto G et al. Intolerance of cow's milk and chronic constipation in children. N Engl J Med 1998; 339: $1100-1104$

5 Read NW. Food and hypersensitivity in functional dyspepsia. Gut 2002; $51: 01 i 50-i 53$

6 Bock SA, Munoz-Furlong A, Sampson HA. Fatalities due to anaphylactic reactions to foods. J Allergy Clin Immunol 2001; 107: $191-193$
7 Young E, Stoneham MD, Petruckevitch A et al. A population study of food intolerance. Lancet 1994; 343: 1127 - 1130

8 Nowak-Wegrzyn A, Conover-Walker MK, Wood RA. Food-allergic reactions in schools and preschools. Arch Pediatr Adolesc Med 2001; 155: 790-795

9 Schäfer T, Bohler E, Ruhdorfer S et al. Epidemiology of food allergy/food intolerance in adults: associations with other manifestations of atopy. Allergy 2001; 56: 1172 - 1179

10 Zuberbier T, Edenharter G, Worm M et al. Prevalence of adverse reactions to food in Germany - a population study. Allergy 2004; 59: 338 - 345

11 Rona RJ, Keil T, Summers C et al. The prevalence of food allergy: a meta-analysis. J Allergy Clin Immunol 2007; 120: 638 646

12 Zuidmeer L, Goldhahn K, Rona RJ et al. The prevalence of plant food allergies: a systematic review. J Allergy Clin Immunol 2008; 121: $1210-1218$

13 Grundy J, Matthews S, Bateman B et al. Rising prevalence of allergy to peanut in children: Data from 2 sequential cohorts. J Allergy Clin Immunol 2002; 110: 784 - 789

14 Bjorksten B. The epidemiology of food allergy. Curr Opin Allergy Clin Immunol 2001; 1: 225-227

15 Okada H, Kuhn C, Feillet $\mathrm{H}$ et al. The "hygiene hypothesis" for autoimmune and allergic diseases: an update. Clin Exp Immunol 2010; 160: 1 - 9

16 Ege MJ, Mayer M, Normand AC. GABRIELA Transregio 22 Study Group . et al. Exposure to environmental microorganisms and childhood asthma. N Engl J Med 2011; 364: 701 - 709

17 Brandtzaeg PE. Current understanding of gastrointestinal immunoregulation and its relation to food allergy. Ann N Y Acad Sci 2002; 964: 13-45

18 Bischoff SC. Physiological and pathophysiological functions of intestinal mast cells. Semin Immunopathol 2009; 31: 185 205

19 Rook GA, Brunet LR. Microbes, immunoregulation, and the gut. Gut 2005; 54: 317 - 320

20 Wehkamp J, Fellermann K, Herrlinger KR et al. Mechanisms of disease: defensins in gastrointestinal diseases. Nat Clin Pract Gastroenterol Hepatol 2005; 2: 406 - 415 ; Review

21 Bischoff SC, Ulmer FA. Eosinophils and allergic diseases of the gastrointestinal tract. Best Pract Res Clin Gastroenterol 2008; 22: $455-479$

22 Majamaa $\mathrm{H}$, Laine S, Miettinen A. Eosinophil protein $\mathrm{X}$ and eosinophil cationic protein as indicators of intestinal inflammation in infants with atopic eczema and food allergy. Clin Exp Allergy 1999; 29: 1502 - 1506

23 Bischoff SC, Mayer ], Wedemeyer ] et al. Colonoscopic allergen provocation (COLAP): a new diagnostic approach for gastrointestinal food allergy. Gut 1997; 40: 745-753

24 Williams RM, Bienenstock J, Stead RH. Mast cells: the neuroimmune connection. Chem Immunol 1995; 61: 208-235

25 Bischoff SC, Gebhardt T. Role of mast cells and eosinophils in neuroimmune interactions regulating mucosal inflammation in inflammatory bowel disease. Adv Exp Med Biol 2006; 579 : $177-208$

26 Rodriguez J, Crespo JF. Clinical features of cross-reactivity of food allergy caused by fruits. Curr Opin Allergy Clin Immunol 2002; $2: 233-238$ 
27 Vieths S, Scheurer S, Ballmer-Weber B. Current understanding of cross-reactivity of food allergens and pollen. Ann N Y Acad Sci 2002; 964: 47-68

28 Valenta R. The future of antigen-specific immunotherapy of allergy. Nat Rev Immunol 2002; 2: 446-453

29 Jansen SC, van Dusseldorp M, Bottema KC et al. Intolerance to dietary biogenic amines: a review. Ann Allergy Asthma Immunol 2003; 91: 233-240

30 Reese I, Ballmer-Weber B, Beyer K et al. Vorgehen bei Verdacht auf Unverträglichkeit gegenüber oral aufgenommenem Histamin. Allergo J 2012: im Druck

31 Leung DY, Bieber T. Atopic dermatitis. Lancet 2003; 361: $151-160$

32 Chong SU, Worm M, Zuberbier T. Role of adverse reactions to food in urticaria and exercise-induced anaphylaxis. Int Arch Allergy Immunol 2002; 129: 19-26

33 Roberts G, Patel N, Levi-Schaffer $\mathrm{F}$ et al. Food allergy as a risk factor for life-threatening asthma in childhood: a case-controlled study. J Allergy Clin Immunol 2003; 112: 168-174

34 Rothenberg ME. Eosinophilic gastrointestinal disorders (EGID). J Allergy Clin Immunol 2004; 113: 11-28

35 Condemi JJ. Allergic reactions to natural rubber latex at home, to rubber products, and to cross-reacting foods. J Allergy Clin Immunol 2002; 110: 107-S110

36 Fasano A, Catassi C. Current approaches to diagnosis and treatment of celiac disease: an evolving spectrum. Gastroenterology 2001; 120: 636-651

37 Suarez FL, Savaiano DA, Levitt MD. Review article: the treatment of lactose intolerance. Aliment Pharmacol Ther 1995; 9: $589-597$

38 Kelso JM, Connaughton C, Helm RM et al. Psychosomatic peanut allergy. J Allergy Clin Immunol 2003; 111: 650-651

39 Bhat K, Harper A, Gorard DA. Perceived food and drug allergies in functional and organic gastrointestinal disorders. Aliment Pharmacol Ther 2002; 16: 969-973

40 Boyce JA, Assa'ad A, Burks AW et al. Guidelines for the diagnosis and management of food allergy in the United States: report of the NIAID-sponsored expert panel. J Allergy Clin Immunol 2010; 126 : 061 -S58

41 Burks AW, Jones SM, Boyce JA et al. NIAID-sponsored 2010 guidelines for managing food allergy: applications in the pediatric population. Pediatrics 2011; 128: 955 - 965
42 Centre for Clinical Practice at NICE (UK). Food Allergy in Children and Young People: Diagnosis and Assessment of Food Allergy in Children and Young People in Primary Care and Community Settings. London: National Institute for Health and Clinical Excellence (UK); 2011

43 Kleine-Tebbe J, Ballmer-Weber B, Beyer K et al. In-vitro-Diagnostik und molekulare Grundlagen von IgE-vermittelten Nahrungsmittelallergien. Allergo J 2009; 18: 132 - 146

44 Niggemann B, Beyer K, Erdmann S et al. Standardisierung von oralen Provokationstests bei Verdacht auf Nahrungsmittelallergie. Allergo J 2011; 20: 149-160

45 Lembcke B. Breath tests in intestinal diseases and functional gastrointestinal diagnosis. Schweiz Rundsch Med Prax 1997; 86: $1060-1067$

46 Simren M, Stotzer PO. Use and abuse of hydrogen breath tests. Gut 2006; 55: 297-303

47 Edwards AM. Oral sodium cromoglycate: its use in the management of food allergy. Clin Exp Allergy 1995; 25 : $0131-33$; Review

48 Joint Task Force on Practice Parameters; American Academy of Allergy, Asthma and Immunology; American College of Allergy, Asthma and Immunology; Joint Council of Allergy, Asthma and Immunology. The diagnosis and management of anaphylaxis: an updated practice parameter. J Allergy Clin Immunol 2005; 115: 03S483-S523

49 Kalliomaki M, Salminen S, Poussa T et al. Probiotics and prevention of atopic disease: 4-year follow-up of a randomised placebo-controlled trial. Lancet 2003; 361: 1869-1871

50 Osborn DA, Sinn JK. Probiotics in infants for prevention of allergic disease and food hypersensitivity. Cochrane Database Syst Rev 2007; 17: CD006475

51 Grüber C, Wendt M, Sulser C et al. Randomized, placebo-controlled trial of Lactobacillus rhamnosus GG as treatment of atopic dermatitis in infancy. Allergy 2007; 62: 1270 - 1276

52 Rose MA, Stieglitz F, Köksal A et al. Efficacy of probiotic Lactobacillus GG on allergic sensitization and asthma in infants at risk. Clin Exp Allergy 2010; 40: 1398 - 1405

53 Leung DY, Sampson HA, Yunginger JW et al. Effect of anti-IgE therapy in patients with peanut allergy. N Engl J Med 2003; 348: $986-993$ 


\section{CME。thieme.de}

\section{CMEFeellahme \\ Viel Erfolg bei lhrer CME-Teilnahme unter http://cme.thieme.de \\ - Diese Fortbildungseinheit ist 12 Monate online für eine CME-Teilnahme verfügbar. \\ - Sollten Sie Fragen zur Online-Teilnahme haben, unter http://cme.thieme.de/hilfe finden Sie eine ausführliche Anleitung.}

Welche Aussage zu Hygiene und Allergien ist richtig?

A Die Hygienetheorie besagt, dass größtmögliche häusliche Hygiene im Kindesalter vor Allergien schützt.

B Studien zur „Hygienetheorie“ konnten zeigen, dass das angeborene Immunsystem für die Ausbildung allergischer Erkrankungen keine Bedeutung hat.

C Laut Hygienetheorie ist eine ausgeprägt hygienische Umgebung ein Risikofaktor für Allergien, schützt aber vor Autoimmunerkrankungen.

D Epidemiologische Studien weisen darauf hin, dass ein gesteigerter Hygienestatus in den Industrienationen mit einer gesteigerten Allergieinzidenz assoziiert ist.

E Exposition mit bakteriellem Lipopolysaccharid in den ersten Lebensjahren führt gehäuft zu Tierhaarallergien.

2 Welche Aussage zu allergischen Reaktionen ist falsch?

A Allergische Reaktionen auf Nahrungsmittel sind meist lgE-vermittelt.

B Immunologische Reaktionen gegen Nahrungsmittel können auch auf gemischten IgE-abhängigen und IgE-unabhängigen Reaktionen beruhen.

C Saisonaler Rhinitis, Urtikaria und atopischem Ekzem liegt in der Regel eine Typ-IV-Reaktion zugrunde.

D An einer allergischen Reaktion in voller Ausprägung sind neben Mastzellen meist auch Granulozyten und Th2-Lymphozyten beteiligt.

E Nach Coombs und Gell werden immunologische Hypersensitivitätsreaktionen in Abhängigkeit vom antigenerkennenden Molekül in 4 Typen eingeteilt.

3 Welche Aussage zu Nahrungsmittelintoleranzen ist richtig?

A Nahrungsmittelintoleranzen und Nahrungsmittelallergien können sowohl pathophysiologisch als auch klinisch klar voneinander abgegrenzt werden.

B Die unspezifische Mastzellaktivierung wird ausschließlich durch Nahrungszusatzstoffe wie Salizylate und Benzoat hervorgerufen.

C Histamin ist der Auslöser des sog. „Chinarestaurant-Syndroms“.

D Intoleranzen gegen biogene Amine wie Tyramin und Glutamat werden auch „Pseudoallergie“ genannt.

E Bei der Laktoseintoleranz kommt es aufgrund mangelnder Laktase im Bürstensaum der Dünndarmepithelien zur bakteriellen Fermentierung der Laktose im Dickdarm.

4 Welche Aussage ist richtig? Die Prävalenz der Nahrungsmittelallergie...
A beträgt bei Kindern 4-8\% und bei Erwachsenen 1-2\%.
B beträgt bei Kindern und Erwachsenen ca. 5\%.
C beträgt bei Erwachsenen $10 \%$ und bei Kindern $5 \%$.
D hat in den letzten Jahrzehnten deutlich abgenommen.
E beträgt bei Kindern 1-2\% und bei Erwachsenen 4-8\%. 


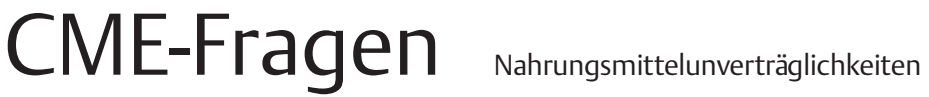
A Neurodermitis
B Otitis serosa
C primär sklerosierende Cholangitis
D orales Allergiesyndrom
E Zöliakie
A Anaphylaktische Reaktionen kommen bei Nahrungsmittelallergien, nicht jedoch bei Nahrungsmittelintoleranzen vor.
B Die Prävalenz der Erdnussallergie, die potenziell zu einer fatalen Anaphylaxie führen kann, beträgt in den USA und UK bei den Erwachsenen ca. 15\%.
C Fatale Anaphylaxie kann schon durch geringste Allergenmengen ausgelöst werden, z. B. beim Küssen.
D Die Nahrungsmittelallergie gilt als Hauptursache für Anaphylaxie in Industrieländern wie USA und Europa.
E Anaphylaxie kann durch Kontakt mit bestimmten Allergenen und gleichzeitige körperliche Anstrengung ausgelöst werden.

\section{Welche Aussage zur Diagnostik von Nahrungsmittelallergien ist falsch?}
A Die sorgfältige Anamnese hinsichtlich Nahrungsmitteln, die nicht vertragen werden, und spezifischen Symptomen ist die Basis der Diagnostik.
B Die Ausschlussdiagnostik muss bei Kindern wesentlich umfangreicher sein als bei Erwachsenen.
C Viele Extrakte von Nahrungsmittelallergenen, die zum Pricktest verwendet werden, weisen ungenügende Standardisierung und Stabilität auf.
D Nahrungsmittelallergien im GI-Trakt werden durch lokal produziertes IgE vermittelt, weshalb der IgE-Serumspiegel bei Nahrungsmittelallergie normal sein kann.
E In unklaren Fällen ist ein kontrollierter Provokationstest für die Diagnosestellung einer Nahrungsmittelallergie erforderlich.

\section{Welche Aussage zur Therapie von Nahrungsmittelallergien ist richtig?}

A Für die Verwendung von Probiotika zur Prävention von Nahrungsmittelallergien gibt es seit einigen Jahren eine verbindliche Empfehlung.

B Eine Eliminationsdiät kann von den Betroffenen nach Lesen eines entsprechenden Merkblattes problemlos umgesetzt werden.

C Nahrungsmittel mit besonders hohem Allergiepotenzial sollen möglichst bald in den Speiseplan von Risiko-Babys eingeführt werden, um eine Toleranz zu erreichen.

D Für eine ergänzende medikamentöse Therapie kommen nur Kortikosteroide in Betracht, da magen-/darmtaugliche Präparationen von Cromoglicinsäure nicht zur Verfügung stehen.

E Patienten mit Anaphylaxie-Anamnese müssen stets mit einem sog. Notfallset an Medikamenten (Adrenalin, Steroid, Antihistaminikum) ausgerüstet werden. 


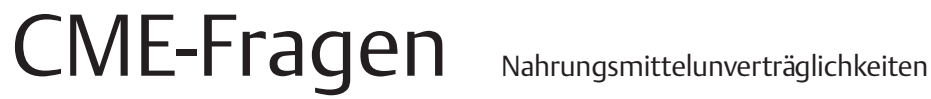

9 Welcher der nachfolgenden Tests kommt nicht im Rahmen der Abklärung von Nahrungsmittelallergien zum Einsatz?
A RAST (Radio-Allergo-Sorbent-Test)
B „double-blind placebo-controlled food challenge“ (DBPCFC)
C Pricktest
D Schilling-Test
E Messung von Eosinophilen-Mediatoren (ECP, EPX) im Serum

10 Welche der nachfolgend genannten Manifestationsformen einer Nahrungsmittelallergie wird meist durch Kreuzreaktionen von Nahrungsmitteln mit Pollen (insbesondere von Birken und Beifußgewächsen) ausgelöst?
A Latex-Nahrungsmittelallergie-Syndrom
B Zöliakie
C intestinales Allergiesyndrom
D orales Allergiesyndrom
E eosinophile Gastroenterokolitis 